\title{
The Global Surgery Research Unit of the Latin American Indicator Research Collaboratory: An Action Model for Grassroots Collection of Lancet Commission on Global Surgery Indicators
}

\section{Elizabeth Elaine Ginalis}

Rutgers New Jersey Medical School https://orcid.org/0000-0002-6097-9017

\section{Carlos Morales}

Universidad de Antioquía: Universidad de Antioquia

\section{Clara Mendoza}

Universidad de Antioquía: Universidad de Antioquia

Joseph S Hanna

Rutgers Robert Wood Johnson Medical School

Gregory L Peck ( $\square$ peckgr@rwjms.rutgers.edu )

Rutgers Robert Wood Johnson Medical School

\section{Research}

Keywords: General Surgery, Global Health, Methods, Public Health Systems Research

Posted Date: May 5th, 2021

DOI: https://doi.org/10.21203/rs.3.rs-476642/v1

License: (c) (i) This work is licensed under a Creative Commons Attribution 4.0 International License.

Read Full License 


\section{Abstract}

Background. The Lancet Commission on Global Surgery (LCOGS) recommends collection of six surgical indicators to inform surgical system strengthening and improve access to surgical care. However, a standardized practice has yet to be described to guide nations towards this endeavor via a sustainable and reproducible grassroots intervention (i.e., developing information management).

Methods. A research team structure, termed a "global surgery research unit" (GSRU), was developed between a hospital in Medellin, Colombia and Rutgers-Robert Wood Johnson Medical School. The GSRU implemented a grassroots intervention in a single center over three months to study the feasibility of obtaining LCoGS indicators 1-4 via hospital medical records, administrative surveys, and patient interviews. The intervention was then retrospectively evaluated utilizing a review of implementation outcomes.

Results. During the feasibility study period, $84.5 \%$ of general/ trauma surgery patients interviewed reported traveling less than two hours. The workforce includes 15 general surgeons, 34 anesthesiologists, and 9 obstetrician-gynecologists. Total surgical volume is estimated to be 15,180 procedures per year, with a 30-day postoperative mortality rate of $1.8 \%$. Evaluation of implementation outcomes revealed grassroots, single center collection of LCoGS indicators was feasible. However, increased workforce and time commitment is required for sustainability.

Conclusions. This learner-driven model introduces a feasible grassroots intervention for LCoGS indicators 1-4 collection in a single center. Grassroots interventions may inform practical collection of individual hospital indicators to inform the information management component of a hospital system. However, continued evaluation of the GSRU and methodological processes is needed to optimize model efficacy and expand indicator data collection.

\section{Background}

An estimated five billion people worldwide lack access to timely, safe, and affordable surgical, obstetric, and anesthesia care $(1,2)$. This burden is disproportionately greater in low- and middle-income countries (LMICs) $(1,3)$. The Lancet Commission on Global Surgery (LCoGS) proposed six core surgical indicators which may be used to characterize surgical systems and inform strengthening $(1,4)$. Collection, followed by analysis and interpretation of these indicators is designed to describe surgical system preparedness, service delivery, and cost impact (Table 1)(2). 
Table 1

Surgical Indicators, Methodology, and Results

\begin{tabular}{|c|c|c|c|c|}
\hline Indicator & $\begin{array}{l}\text { LCoGS Indicator } \\
\text { Description }\end{array}$ & $\begin{array}{l}\text { Hospital Specific } \\
\text { Indicator }\end{array}$ & Methods & Results \\
\hline $\begin{array}{l}1 \\
\text { (Preparedness) }\end{array}$ & $\begin{array}{l}\text { 2-hour access to } \\
\text { hospitals that can } \\
\text { provide Bellwether } \\
\text { Procedures } \\
\text { (Laparotomy, C- } \\
\text { section, Open } \\
\text { Fractures) }\end{array}$ & $\begin{array}{l}\text { Percentage of } \\
\text { patients that } \\
\text { arrive to HUSVF } \\
\text { within } 2 \text { hours }\end{array}$ & $\begin{array}{l}\text { Patient } \\
\text { interviews with } \\
\text { general surgery, } \\
\text { trauma surgery, } \\
\text { and OBGYN } \\
\text { patients }\end{array}$ & $\begin{array}{l}84.5 \% \text { of general } \\
\text { and trauma } \\
\text { surgery patients \& } \\
73.2 \% \text { of OBGYN } \\
\text { patients arrived } \\
\text { within } 2 \text { hours }\end{array}$ \\
\hline $\begin{array}{l}2 \\
\text { (Preparedness) }\end{array}$ & $\begin{array}{l}\text { Surgeon, } \\
\text { Anesthesiologist, } \\
\text { OBGYN (SAO) } \\
\text { density }\end{array}$ & $\begin{array}{l}\text { Number of } \\
\text { surgeons, } \\
\text { anesthesiologists, } \\
\text { and OBGYNs at } \\
\text { HUSVF }\end{array}$ & $\begin{array}{l}\text { Hospital } \\
\text { administrative } \\
\text { survey }\end{array}$ & $\begin{array}{l}15 \text { general } \\
\text { surgeons, } 34 \\
\text { anesthesiologists, } \\
\& 8 \text { OBGYNs }\end{array}$ \\
\hline $\begin{array}{l}3 \\
\text { (Delivery) }\end{array}$ & Surgical volume & $\begin{array}{l}\text { Number of } \\
\text { surgical } \\
\text { operations } \\
\text { performed } \\
\text { annually at } \\
\text { HUSVF }\end{array}$ & $\begin{array}{l}\text { Retrospective } \\
\text { data from } \\
\text { hospital medical } \\
\text { records for all } \\
\text { surgical patients }\end{array}$ & $\begin{array}{l}15,180 \text { surgeries } \\
\text { per year }\end{array}$ \\
\hline $\begin{array}{l}4 \\
\text { (Delivery) }\end{array}$ & $\begin{array}{l}\text { In-hospital 30-day } \\
\text { postoperative } \\
\text { mortality rate (non- } \\
\text { risk adjusted) }\end{array}$ & $\begin{array}{l}\text { In-hospital 30-day } \\
\text { postoperative } \\
\text { mortality rate }\end{array}$ & $\begin{array}{l}\text { Retrospective } \\
\text { data from } \\
\text { hospital medical } \\
\text { records for all } \\
\text { patients } \\
\text { undergoing } \\
\text { surgery at } \\
\text { HUSVF }\end{array}$ & $\begin{array}{l}\text { Postoperative } \\
\text { mortality rate of } \\
1.8 \%\end{array}$ \\
\hline $\begin{array}{l}5 \\
\text { (Cost Impact) }\end{array}$ & $\begin{array}{l}\text { Risk of } \\
\text { impoverishing } \\
\text { expenditure from } \\
\text { surgical care }\end{array}$ & $\begin{array}{l}\text { Percentage of } \\
\text { patients suffering } \\
\text { from } \\
\text { impoverishing } \\
\text { expenditure from } \\
\text { surgical care }\end{array}$ & None & None \\
\hline $\begin{array}{l}6 \\
\text { (Cost Impact) }\end{array}$ & $\begin{array}{l}\text { Risk of catastrophic } \\
\text { expenditure from } \\
\text { surgical care }\end{array}$ & $\begin{array}{l}\text { Percentage of } \\
\text { patients suffering } \\
\text { from catastrophic } \\
\text { expenditure from } \\
\text { surgical care }\end{array}$ & None & None \\
\hline
\end{tabular}

Four of these six core surgical indicators have since been adopted by the World Bank as World Development Indicators. This milestone in the effort to assess and improve the surgical system globally has focused international attention and aligned national priorities through a common discussion(4-7). Additional advocacy by the World Health Organization (WHO) through the World Health Assembly Resolution 68.15 has encouraged this data collection to support the development and implementation of National Surgical, Obstetric, and Anesthesia Plans (NSOAPs)(8). Following NSOAP development and 
implementation, iterative collection of these surgical indicators may facilitate continuous downstream evaluation and monitoring(9).

Although LCoGS proposed these core indicator metrics with targets, no specific guidance was provided on how to start and iteratively improve grassroots collection of indicator data in settings without coordinated health system information management mechanisms. To globally report these indicators to the World Bank, a sufficiently detailed, sustainable, and cost-effective methodology to achieve timely data collection is necessary. Current efforts to collect LCoGS indicator data rely on top-down approaches, requiring involvement of Ministries of Health, national healthcare informatics and database management, and hospital directors interested in population metrics extrapolated from grassroots levels of care, i.e., clinical settings $(5,10-14)$. Although top-down methods enable collection of large volume data, incomplete documentation, fragmented data sources, and exclusion of small hospitals during national data sampling may compromise the accuracy and generalizability of results. Additionally, measurement and selection bias may be introduced, particularly in LMICs with variable health information management system technology $(5,10,15)$. As such, a facility-level analysis of hospital surgical capabilities to accurately collect core surgical indicator data is needed, particularly in small hospitals which may otherwise be under-represented in a national evaluation. Additionally, grassroots methods may also inform health system performance specific to each hospital via continuous data surveillance of these indicators to inform processes such as quality improvement, budget planning, resource allocation, and training needs.

Presently there is a lack of literature describing systematic grassroots collection of LCoGS indicators and a link to national agenda. Ideally a proposed grass-roots data collection effort should be objectively evaluated for its efficacy, effectiveness, and equity. Dissemination and implementation (D\&I) research methods are well suited to assess intervention processes using described implementation outcome variables and process appropriate frameworks(16-18). Implementation science has been used successfully to objectively characterize, guide, or evaluate contemporary interventions in health services research and academic global surgery(16-22). Appropriately chosen D\&I frameworks enable identification of barriers and facilitators to an intervention (i.e., protocol implementation success) through a systematic process, structure, and outcome assessment(16). Proctor and colleagues described eight implementation outcomes (acceptability, adoption, appropriateness, cost, feasibility, fidelity, penetration, and sustainability) for the systematic assessment of intervention efficacy and effectiveness (Table 2). To date, D\&I methodologies have yet to be applied to assess and improve the process and structure of LCoGS indicator data collection, analysis, and interpretation. 
Table 2

Implementation Variables and Definitions

\begin{tabular}{|c|c|}
\hline $\begin{array}{l}\text { Implementation } \\
\text { Variable }\end{array}$ & Definition \\
\hline Acceptability & $\begin{array}{l}\text { Perception among stakeholders that a given treatment, service, practice, or } \\
\text { innovation is agreeable or satisfactory }\end{array}$ \\
\hline Adoption & The intention or action to employ an innovation \\
\hline Appropriateness & $\begin{array}{l}\text { The perceived fit, relevance, or compatibility of the innovation for a given practice } \\
\text { setting; and/or perceived fit of the innovation to address a particular problem }\end{array}$ \\
\hline Cost & The cost impact of an implementation effort \\
\hline Feasibility & $\begin{array}{l}\text { The extent to which a new innovation can be successfully carried out within a } \\
\text { given setting }\end{array}$ \\
\hline Fidelity & $\begin{array}{l}\text { The degree to which an intervention was implemented as it was prescribed in the } \\
\text { original protocol or as it was intended by the program developers }\end{array}$ \\
\hline Penetration & The integration of a practice within a service setting \\
\hline Sustainability & $\begin{array}{l}\text { The extent to which a newly implemented intervention is maintained within a } \\
\text { setting's ongoing operations }\end{array}$ \\
\hline
\end{tabular}

In this study, we describe and evaluate a participatory action, team-based global surgery research model in Medellin, Colombia for grassroots LCoGS core surgical indicators 1-4 data collection. This model was qualitatively evaluated using D\&I methods to determine efficacy and effectiveness using Proctor's implementation outcomes framework to identify promotors and barriers for iterative design improvement $(16,17)$.

\section{Methods}

\section{Global Surgery Research Team Model and Setting}

The Latin American Indicator Research Collaboratory (LAIRC) was created to advance development of a cost-effective, reproducible, and standardized methodology for surgical system strengthening at the grassroots level between North America and Latin America and the Caribbean(23). Foundational to the LAIRC is a participatory action research and team-based "twinning" partnership between a LMIC and a high-income country (HIC). Each unit of a twinned pair has been termed a global surgery research unit (GSRU), composed of a research fellow(s) and at least one faculty member. The overarching strategy is for the HIC GSRU to partner with the low-income country/ middle-income country (LIC/MIC) GSRU to understand local needs and co-develop research programs and appropriate solutions led by the LIC/MIC.

In June 2017, a Colombian GSRU was established at a medical school affiliated, private, non-profit, tertiary hospital with 662 patient beds and 15 operating rooms in Medellin, Colombia. A sister GSRU was 
simultaneously established at Rutgers-Robert Wood Johnson Medical School (RWJMS) in New Brunswick, New Jersey. Both institutions completed a Memorandum of Understanding documenting the academic relationship(24). This created a network composed of hospital, community, and student members who were engaged throughout all stages of the research process and were stakeholders for indicator data collection, emphasizing the community-based participatory approach of the GSRU model $(25,26)$. These MIC and HIC GSRUs worked together to collect LCoGS core surgical indicator 1-4 data using a transnational protocol that was adapted based on iterative input and participation. The shared research protocol was approved by both institutional review boards.

\section{Data Collection}

The research protocol employed mixed methods consisting of patient interviews, hospital leadership interviews, and repurposing data from existing hospital databases for LCoGS indicator collection. Definitions of variables related to surgical indicators $1-4$, research methods, and results are summarized in Table 1. Team members from the Rutgers GSRU travelled to Medellin in June 2017 to participate in surgical indicator and patient demographic data collection.

\section{Methods for Surgical Indicator Data Collection}

Patient Demographics. Surgical and obstetrics/gynecology patient demographic data were collected for a one-month period via daily in-person patient interviews during morning rounds (Additional file 1).

Time to Hospital. Daily in-person patient interviews of general surgery, trauma surgery, and obstetrics/gynecology patients were conducted to collect data regarding transport time to the study facility. Newly admitted patients that underwent an operation on admission from these services were rounded on daily following their operation (Additional file 1).

Surgeon, Anesthesiologist, and Obstetrician (SAO) Complement. In-person, semi-structured interviews, adapted from the World Health Organization Hospital Assessment Tool (HAT), were conducted with hospital leaders (Additional file 2)(27). These data were used to determine the total number of surgeons, anesthesiologists, and obstetricians at the study facility.

Surgical Volume. Total surgical volume across all surgical specialties during the month of June 2017 was determined retrospectively from a pre-existing hospital administrative database.

Postoperative Mortality. For the month of June in 2017, all cause, non-risk adjusted, in-hospital, postoperative mortality data were collected retrospectively from a pre-existing hospital administrative database.

\section{Implementation Outcomes Analysis}

The GSRU model and data collection intervention processes were retrospectively analyzed using a questionnaire (Table 3) that guided in-person, semi-structured interviews with Colombian GSRU stakeholders regarding the June 2017 experience. Interview questions were developed following the 
conclusion of the 2017 experience and categorized into implementation outcomes based on the framework described by Proctor et al., describing acceptability, adoption, appropriateness, cost, feasibility, fidelity, penetration, and sustainability (16). Throughout the June 2017 experience, informal feedback was also recorded from interactions with Latin American stakeholders, including patients, students, physicians, and hospital administrators, and during weekly team meetings. This formal and informal feedback was similarly analyzed to identify barriers and facilitators to the GSRU implementation and data collection interventions. 
Table 3

Implementation Outcomes Questionnaire

\section{Implementation Question \\ Outcome}

Acceptability Were you satisfied with the data collection process?

Did you agree with the process that was taken to obtain approval for the project?

Did you agree with the process that was taken to form a GSRU for data collection?

Adoption

Was the implementation of the GSRU for data collection adopted well by the hospital and staff?

What initial thoughts did you have when first approached about this project?

How have these thoughts changed after implementation of the GSRU to collect indicator data?

Appropriateness Do you feel that the GSRU with multiple team members from both HIC and LMIC systems was the best way to collect data?

Did this intervention fit your system?

What other support would increase data collection?

Do you have recommendation for an improved system based on how your hospital system is structured?

Cost

What costs were incurred during the data collection process?

Were these costs more or less than expected?

Do you feel these costs may be a barrier to future data collection in other hospitals in Colombia?

Feasibility Do you feel GSRU is feasible to collect indicator data?

Did the GSRU structure fit well within your hospital system to collect indicator data?

How can we improve the structure of the GSRU to improve data collection of all indicators simultaneously?

Fidelity

Did the GSRU collect indicator data in the methods approved by ethics committee?

What modifications were made to the process in order to improve the amount of data that was collected?

What future improvements do you suggest to improve GSRU data collection?

Penetration

Was there difficulty with any plans in integrating with hospital system where indicator data collection occurred? 


\section{Implementation Question}

Outcome

Could the GSRU data collection process be integrated into hospital system where data is continually collected?

What steps are necessary to accomplish this continual data collection?

Sustainability What suggestions do you have to make indicator data sustainable at your institution?

Are cost, human workforce, and resources barriers to achieving implementation of sustainability?

\section{Results}

Men comprised $71.9 \%$ of the general and trauma surgery patients undergoing operative care, with a mean age of 40.2 years (SD 20.3 years). The mean age of OBGYN patients was 27.9 years (SD 8.9 years). The distribution of transportation modalities for general and trauma surgery patients was ambulance (42.8\%), taxi $(28.3 \%)$, other $(16.7 \%)$, private car $(8.9 \%)$, and by foot $(3.3 \%)$. In contrast, the distribution of transportation modalities among OBGYN patients was ambulance (73.6\%), taxi (11.3\%), car (9.4\%), and other (5.7\%). "Other" forms of transportation include metro/subway, bus, or police car.

The majority of general and trauma surgery patients (84.5\%) as well as OBGYN patients $(73.2 \%)$ arrived to the emergency department within two hours, with an average patient-reported time of 77.9 minutes (SD 145.5 minutes). For patients who were transferred from another hospital (referred to as "interfacility transfer"), the time estimate only included final interfacility transport time. Excluding patient data from interfacility transfers, $82.9 \%$ of general and trauma surgery patients and $82.6 \%$ of OBGYN patients arrived to the emergency room from the initial site of origin within two hours.

In response to structured interviews using the WHO hospital assessment tool, hospital leadership indicated that the physician workforce included 15 general surgeons, 34 anesthesiologists, and 9 obstetrician/gynecologists. This SAO workforce performed 1,265 operative procedures in June 2017, which corresponds to an estimated 15,180 surgeries annually (1265 procedures $\times 12$ months).

Of the 1,265 total procedures performed during the study period, an observed non-risk adjusted inhospital postoperative mortality rate of $1.8 \%(n=23$ deaths). Two of the 23 deaths $(8.7 \%)$ occurred following a laparotomy. No postoperative deaths occurred in patients undergoing cesarean section or open fracture treatment. General and trauma surgery had the highest mortality with 11 deaths $(47.8 \%)$, followed by neurosurgery (17.4\%) and vascular surgery (17.4\%) with 4 deaths each.

\section{Evaluation of Implementation Outcomes}

Analysis of the GSRU intervention is summarized using the implementation variables in Table 4. With respect to acceptability, GSRU stakeholders found the research protocol to be satisfactory. Latin 
American partners were motivated, informed, and readily formed relationships with hospital leaders and research fellows. However, lack of Latin American medical students' integration in the team was perceived as a barrier to implementation due to the need for a more robust research workforce. One Latin American clinical faculty member from the GSRU stated, "It is very important for [Colombian] students here to be participating in the local story" to best advocate for patients in their community.

Table 4

Summary of Implementation Outcomes

\begin{tabular}{|ll|}
\hline $\begin{array}{l}\text { Implementation } \\
\text { Variable }\end{array}$ & Outcomes \\
\hline Acceptability & $\begin{array}{l}\text { - Protocol approved by ethics committee at University of Antioquia and IRB } \\
\text { committee at Rutgers University } \\
\text { - Protocol for collection of LCoGS indicators 1-4 deemed appropriate } \\
\text { - University of Antioquia medical students recruited to GSRU for 2018 } \\
\text { implementation period }\end{array}$ \\
\hline Adoption & - Protocol successfully adopted in 2017 \\
\hline Appropriateness & $\begin{array}{l}\text { - Self-reported estimates during patient interviews may be inaccurate (especially } \\
\text { indicator 1) }\end{array}$ \\
\hline Costs & $\begin{array}{l}\text { - Direct costs covered by exogenous funding } \\
\text { - Indirect costs not measured }\end{array}$ \\
\hline Feasibility & - Cost not perceived to be a barrier to implementation \\
\hline Penetration & - Burden of data collection is large \\
\hline Sustainability & - Protocol was closely adhered to as prescribed \\
\hline
\end{tabular}

Appropriateness. Overall, both US and Colombian GSRU members felt the intervention to be appropriate, though it could be improved for indicator 1 . They recognized that methods for indicator 1 may introduce recall bias during patient interviews. Additionally, some patients were transferred from other hospitals, and this interfacility transport time was captured during the patient interviews. However, we did not obtain transport time from incident location to the original hospital in these patients. The sum of these transport times is the true indicator 1 value. 
Costs. The direct costs for the North American medical students and general surgery resident to travel and board in Colombia were funded entirely by the U.S. Agency for International Development-Research Innovation Fellowship Program (USAID-RIFP), with a scholarship award of approximately $\$ 2500$ per student for the month. There were no additional direct costs associated with data collection. Indirect costs were identified as a barrier to long-term implementation of the intervention. However, the indirect costs, including determination of the cost of Latin American clinical faculty absence from clinical responsibilities due to involvement in surgical indicator data research, were not specifically measured.

Feasibility. The protocol required a significant time commitment for GSRU research partners to collect data prospectively throughout the year due to the absence of Colombian medical student participation. When the GSRU teams were working together in tandem, the intervention was most feasible. The protocol requires assistance from and collaboration with hospital leaders to promote longitudinal data collection as well as with medical students to conduct daily interviews with patients (158 total patients interviewed, requiring approximately 15 minutes per patient), representing a significant unfunded time burden for both sister GSRUs and members. Additionally, each interview with the hospital leaders took approximately 15 minutes.

Fidelity. The protocol was closely adhered to as originally developed. GSRU members stated that the research protocol could be modified to fit a range of hospital settings in Colombia, particularly those with hospital databases.

Penetration. This intervention led to collection of baseline data of LCoGS indicators 1-4 while in country. The protocol was successfully integrated in the hospital setting while Rutgers GSRU members were presented in Medellin but failed to penetrate the hospital system following their departure.

Sustainability. GSRU members stated they were able to execute the research protocol in the short term while the two teams were working together. However, due to lack of sufficient workforce and formal Colombian medical student engagement and participation, data collection ceased following the departure of North American partners.

\section{Discussion}

A standardized methodology for collecting surgical indicator data, now mandated by the World Health Organization, is necessary to inform the global surgical system situation analysis effort to address profound barriers in access to surgical care(4). Historically, efforts in LICs and MICs have focused on augmenting the health system domains of workforce, service delivery, and finance $(15,28-31)$. To our knowledge, the present study is the first reported effort to quantitatively and qualitatively assess a prospectively designed grassroots data collection intervention for LCoGS indicators 1-4. Our study suggests that this approach, predicated on a participatory action-based twinned-team model between academic units in an LIC/MIC and HIC, can be successfully implemented in an individual facility. Whereas the majority of the literature to date has focused on indicator efforts in Africa and Asia, this is the first directed grassroots effort to assess LCoGS indicator collection in Latin America. 
Quantitatively, the team successfully collected data with respect to SOA system preparedness. In this busy urban academic bellwether capable facility, $83 \%$ of general/ trauma surgery and OBGYN patients reported a mean transport time within two hours (indicator 1) if inter-facility transportation was not required. However, $32.5 \%$ of surveyed patients required interfacility transportation for appropriate services, raising concern that a significant proportion of the population is outside of the 2 hour-access target set by the LCoGS. This is consistent with previous findings that between approximately $15-30 \%$ of the Colombian population cannot readily access a bellwether capable facility within 2 hours $(14,22)$. Regarding indicator 2, we were able to accurately determine the number of general surgeons, anesthesiologists, and obstetricians/gynecologists. While a true SAO density cannot be calculated because the catchment area for any given facility is not homogenous, in aggregate, this type of data can be used to determine indicator 2 at the municipal or departmental level.

In addition to preparedness, SOA system service delivery was assessed, and annual performance extrapolated. To begin to characterize indicator 3 , the team identified 1265 operative procedures performed during the study month. Annualized, this corresponds to a total operative volume of 15,180 procedures. Although this projection is approximately $9 \%$ less than the actual volume performed $(16,710$ surgical procedures in 2017), this is an acceptable variance given the short sampling period. During the study period, we calculated an all-cause, non-risk adjusted 30-day, in-hospital postoperative mortality rate (POMR) of $1.8 \%$ (indicator 4 ). While this single center rate is 1.7 -fold higher than the aggregate mean for the city of Medellin reported in 2016, it is within the range of $0.02 \%-4.78 \%$ reported across the 185 municipalities with observed post-operative deaths within 30-days. Furthermore, although this observed POMR is 2.4 fold greater than the reported Colombian median in $2016(0.74 \%)(22)$, it is consistent with the observation that an increased POMR is correlated with areas of higher socioeconomic status and SAO density such as Medellin $(10,22)$. While the underlying reason for this observation remains unexplored, the present estimate is subject to sampling bias given the short study period and case-mix index.

Qualitatively, we used Proctor's implementation outcomes framework to objectively evaluate our team construct (GSRU) and protocol for LCoGS indicator data collection. GSRU data collection activities were successfully performed by learners (medical students) with both local and remote academic faculty mentorship. Noteworthy, though not addressed in the present analysis, is the benefit to learners of cultural immersion, access to local mentorship, and international relationship development for future career growth. In this pilot, our Latin American partners did not incur any direct costs as this was borne by the USAID grant and HIC students themselves. Given the demonstrated success with which learners were able to navigate the hospital system and implement data collection protocols, it is anticipated that Colombian learner engagement would allow for career growth while minimizing future direct costs in the future. While this does not address future HIC learner travel expenses (estimated at $\$ 2500 /$ learner/month), in balance, when considered through the lens of the average per credit hour education cost to learners in the US (\$1537/hour) these direct costs are commensurate with the nototherwise available, unique, and now mandatory LCME/AAMC education experience(32). Nonetheless, identification of exogenous funding and cost sharing is an important consideration for long term 
sustainability of the program. Thus, this is a cost-effective model for initiation of bi-national indicator research with respect to HIC incurred direct expenses. Notwithstanding, the impact of the direct costs incurred, assessment of indirect costs is particularly applicable for the Latin American faculty component of the GSRU as their participation came at the expense of required clinical activities. Local resources to support this aspect of professional development and activity should be identified for future sustainability and growth based on the academic goals of faculty and departmental leadership.

Through the implementation outcomes framework, we identified the need for greater GSRU workforce. The time burden of data collection, specifically patient and family interviews to determine indicator 1 , was significant. The absence of participating Colombian learners in this pilot resulted in significant barriers to feasibility and sustainability of the protocol. However, despite this project's lack of MIC student participation in indicator data collection, the Colombian GSRU has since created a medical student group in collaboration with the organization International Student Surgical Network (InciSioN). This expansion of opportunities to Colombian medical students furthers learner engagement, promotes research, and provides an avenue for global partnerships and action.

There are several limitations in our study. This study was conducted at a single, private, academic bellwether facility. Human and material resource availability, as well as barriers and facilitators to research will differ in other facilities and regions. Consequently, the protocol and GSRU model may need to be adapted according to the resources of the next hospitals that apply the model. Implementation science principles may help to guide the iterative development and documentation of these efforts for dissemination. A second limitation was the short GSRU implementation period of approximately one month which may not be representative of the whole year. The implementation period was restricted due to the HIC medical students' academic calendar, which could only accommodate international travel during the described time period. A lack of student workforce in the Colombian GSRU prevented independent long-term data collection. Therefore, dissemination of education and training of HIC and MIC student participants to achieve greater MIC student involvement and more robust institutional engagement are underway. Gratifyingly, a significant increase in Colombian GSRU medical students' participation has been observed. Third, data collection was restricted to specific patient populations due to established partnerships with the general/ trauma surgery and obstetrics-gynecology services in the host institution. This limited the GSRU's ability to perform data collection across all surgical specialties. Fourth, self-reported estimates of length of time to the facility was subject to recall bias resulting in an unmeasurable time variance as no objective documentation existed against which to verify self-reports. Finally, we were unable to estimate a catchment area for this single hospital to inform an SAO density calculation as there are multiple tertiary hospitals with overlapping service areas in Medellin.

With respect to cost protection, we were unable to assess indicators 5 and 6 in this setting. Previous studies in Africa reporting on indicators 5 and 6 utilized an in-person questionnaire upon a patient's hospital discharge, which was successful as direct medical costs are upfront or otherwise known during hospitalization $(28,29)$. We did not find this protocol to be adequate due to human resource limitations and inability to capture direct costs at time of hospitalization. Work is ongoing to develop a reliable and 
effective survey tool and the research infrastructure with which to accurately assess direct and indirect costs.

\section{Conclusion}

This study introduces a successful grassroots intervention in a single hospital setting for LCoGS indicator 1-4 collection using existing hospital resources and structured stakeholder and patient interviews. Further development of the research workforce recruitment paradigm and interview adaptation is needed for successful longitudinal data collection of indicators 1-6. Such grassroots efforts may inform municipal and facility quality improvement processes. Reciprocal or "twinning" partnerships and cultural proficiency to LICs/MICs research processes, effective community participation, and local championship for research has promoted these early stages of collaborative LCoGS indicator data collection. Methods drawn from implementation outcome evaluation will be continuously utilized to evaluate, monitor, and improve the GSRU model. Establishing local championship (particularly targeting Latin American student research programs) and cross-sectoral involvement (including the Colombian Ministry of Health, Ministry of Education, and medical school programs) is imperative to ensure on-going data collection, analysis and interpretation. By objectively characterizing the facilitators and barriers to success of the GSRU model through the novel application of an implementation framework, this work may inform an iterative process building upon the GSRU incorporating grassroots partnerships in other countries to advance LCoGS indicator informed national public health and surgical health care plans.

\section{Abbreviations}

LMICs: low- and middle-income countries

LCoGS: Lancet Commission on Global Surgery

NSOAPs: National Surgical, Obstetric, and Anesthesia Plans

D\&I: Dissemination and implementation

LAIRC: Latin American Indicator Research Collaboratory

HIC: high-income country

GSRU: global surgery research unit

LIC/MIC: low-income country/ middle-income country

RWJMS: Rutgers-Robert Wood Johnson Medical School

SAO: Surgeon, Anesthesiologist, and Obstetrician

WHO: World Health Organization 
HAT: Hospital Assessment Tool

USAID-RIFP: U.S. Agency for International Development-Research Innovation Fellowship Program

POMR: postoperative mortality rate

InciSioN: International Student Surgical Network

\section{Declarations}

\section{Ethics approval and consent to participate}

The research protocol was approved by the Rutgers Institutional Review Board (Study ID:

Pro20170000334) and the Universidad de Antioquia/ Hospital San Vicente Fundación de Medellín institutional review board equivalent.

\section{Consent for publication}

Not applicable.

\section{Availability of data and materials}

The datasets used and/or analyzed during the current study are available from the corresponding author on reasonable request.

\section{Competing interests}

The authors declare that they have no competing interests.

\section{Funding}

United States Agency for International Development - Research Fellowship Innovation Program

\section{Authors' Contributions}

$\mathrm{CM}, \mathrm{JSH}$, and GLP made substantial contributions to the conception and design of the work. EEG, CM, and CM collected, analyzed, and interpreted the data. EEG drafted the manuscript. JSH and GLP provided substantial revisions to the manuscript. All authors read and approved the final manuscript.

\section{Acknowledgements}

Not applicable.

\section{References}


1. Meara JG, Leather AJ, Hagander L, Alkire BC, Alonso N, Ameh EA, et al. Global Surgery 2030: evidence and solutions for achieving health, welfare, and economic development. Lancet (London, England). 2015;386(9993):569-624.

2. Alkire BC, Raykar NP, Shrime MG, Weiser TG, Bickler SW, Rose JA, et al. Global access to surgical care: a modelling study. The Lancet Global health. 2015;3(6):e316-23.

3. Weiser TG, Regenbogen SE, Thompson KD, Haynes AB, Lipsitz SR, Berry WR, et al. An estimation of the global volume of surgery: a modelling strategy based on available data. Lancet (London, England). 2008;372(9633):139-44.

4. Raykar NP, Ng-Kamstra JS, Bickler S, Davies J, Greenberg SLM, Hagander L, et al. New global surgical and anaesthesia indicators in the World Development Indicators dataset. BMJ global health. 2017;2(2):e000265.

5. Guest GD, McLeod E, Perry WRG, Tangi V, Pedro J, Ponifasio P, et al. Collecting data for global surgical indicators: a collaborative approach in the Pacific Region. BMJ global health. 2017;2(4):e000376.

6. Strategizing national health in the 21st century: a handbook. World Health Organization. 2016.

7. Citron I, Chokotho L, Lavy C. Prioritisation of Surgery in the National Health Strategic Plans of Africa: A Systematic Review. World journal of surgery. 2016;40(4):779-83.

8. Juran S, Gruendl M, Marks IH, Broer PN, Guzman JM, Davies J, et al. The need to collect, aggregate, and analyze global anesthesia and surgery data. Canadian journal of anaesthesia = Journal canadien d'anesthesie. 2019;66(2):218-29.

9. Sonderman KA, Citron I, Mukhopadhyay S, Albutt K, Taylor K, Jumbam D, et al. Framework for developing a national surgical, obstetric and anaesthesia plan. BJS open. 2019;3(5):722-32.

10. Massenburg BB, Saluja S, Jenny HE, Raykar NP, Ng-Kamstra J, Guilloux AGA, et al. Assessing the Brazilian surgical system with six surgical indicators: a descriptive and modelling study. BMJ Glob Health. 2017;2(2):e000226.

11. Bruno E, White MC, Baxter LS, Ravelojaona VA, Rakotoarison HN, Andriamanjato HH, et al. An Evaluation of Preparedness, Delivery and Impact of Surgical and Anesthesia Care in Madagascar: A Framework for a National Surgical Plan. World journal of surgery. 2017;41(5):1218-24.

12. Baxter LS, Ravelojaona VA, Rakotoarison HN, Herbert A, Bruno E, Close KL, et al. An Observational Assessment of Anesthesia Capacity in Madagascar as a Prerequisite to the Development of a National Surgical Plan. Anesthesia and analgesia. 2017;124(6):2001-7.

13. Anderson JE, Ndajiwo AB, Nuhu SA, Lawal OA, Amedu JO, Ameh EA. Assessment of Capacity to Meet Lancet Commission on Global Surgery Indicators in the Federal Capital Territory, Abuja, Nigeria. World journal of surgery. 2019;43(3):704-14.

14. Hanna JS, Herrera-Almario GE, Pinilla-Roncancio M, Tulloch D, Valencia SA, Sabatino ME, et al. Use of the six core surgical indicators from the Lancet Commission on Global Surgery in Colombia: a situational analysis. The Lancet Global Health. 2020;8(5):e699-e710. 
15. Anderson GA, Ilcisin L, Abesiga L, Mayanja R, Portal Benetiz N, Ngonzi J, et al. Surgical volume and postoperative mortality rate at a referral hospital in Western Uganda: Measuring the Lancet Commission on Global Surgery indicators in low-resource settings. Surgery. 2017;161(6):1710-9.

16. Proctor E, Silmere H, Raghavan R, Hovmand P, Aarons G, Bunger A, et al. Outcomes for implementation research: conceptual distinctions, measurement challenges, and research agenda. Administration and policy in mental health. 2011;38(2):65-76.

17. Damschroder LJ, Aron DC, Keith RE, Kirsh SR, Alexander JA, Lowery JC. Fostering implementation of health services research findings into practice: a consolidated framework for advancing implementation science. Implementation science : IS. 2009;4:50.

18. Saluja S, Silverstein A, Mukhopadhyay S, Lin Y, Raykar N, Keshavjee S, et al. Using the Consolidated Framework for Implementation Research to implement and evaluate national surgical planning. BMJ Glob Health. 2017;2(2):e000269.

19. Glasgow RE, Vinson C, Chambers D, Khoury MJ, Kaplan RM, Hunter C. National Institutes of Health approaches to dissemination and implementation science: current and future directions. American journal of public health. 2012;102(7):1274-81.

20. Theobald S, Brandes N, Gyapong M, El-Saharty S, Proctor E, Diaz T, et al. Implementation research: new imperatives and opportunities in global health. Lancet (London, England). 2018;392(10160):2214-28.

21. Curran GM, Bauer M, Mittman B, Pyne JM, Stetler C. Effectiveness-implementation hybrid designs: combining elements of clinical effectiveness and implementation research to enhance public health impact. Med Care. 2012;50(3):217-26.

22. Peck GL, Hanna JS. The National Surgical, Obstetric, and Anesthesia Plan (NSOAP): Recognition and Definition of an Empirically Evolving Global Surgery Systems Science; Comment on "Global Surgery - Informing National Strategies for Scaling Up Surgery in Sub-Saharan Africa". International Journal of Health Policy and Management. 2018:-.

23. Peck G, Saluja S, Blitzer DN, Sarma D, Anderson GA, Rodas E, et al. Using global surgical indicators to improve trauma care in Latin America. Bulletin of the American College of Surgeons. 2017;102(4):116.

24. Latin American Indicator Research Collaboratory 2018 [Available from: http://rutgersglobalsurgery.org/wp-content/uploads/2018/12/Abbrev.LAIRC-Toolkit.pdf.

25. MacDonald $\mathrm{C}$. Understanding participatory action research: A qualitative research methodology option. The Canadian Journal of Action Research. 2012;13(2):34-50.

26. Kelly PJ. Practical suggestions for community interventions using participatory action research. Public health nursing (Boston, Mass). 2005;22(1):65-73.

27. Tobin MK, Geraghty JR, Engelhard HH, Linninger AA, Mehta Al. Intramedullary spinal cord tumors: a review of current and future treatment strategies. Neurosurgical focus. 2015;39(2):E14.

28. Anderson GA, Ilcisin L, Kayima P, Abesiga L, Portal Benitez N, Ngonzi J, et al. Out-of-pocket payment for surgery in Uganda: The rate of impoverishing and catastrophic expenditure at a government 
hospital. PloS one. 2017;12(10):e0187293.

29. Bijlmakers L, Wientjes M, Mwapasa G, Cornelissen D, Borgstein E, Broekhuizen $H$, et al. Out-of-pocket payments and catastrophic household expenditure to access essential surgery in Malawi - A crosssectional patient survey. Ann Med Surg (Lond). 2019;43:85-90.

30. Albutt K, Punchak M, Kayima P, Namanya DB, Anderson GA, Shrime MG. Access to Safe, Timely, and Affordable Surgical Care in Uganda: A Stratified Randomized Evaluation of Nationwide Public Sector Surgical Capacity and Core Surgical Indicators. World J Surg. 2018;42(8):2303-13.

31. Siddiqi S, Khan MS, Rizvi N, Naeem I, Rozi S, Enam A, et al. Are Rural Hospitals in Pakistan Responding to the Global Surgery Movement? An Analysis of the Gaps, Challenges and Opportunities. World J Surg. 2020;44(4):1045-52.

32. Trends in College Pricing 2019: College Board; 2019 [

\section{Supplementary Files}

This is a list of supplementary files associated with this preprint. Click to download.

- Additionalfile1FinalPlanCRunsheetApr242017.docx

- Additionalfile2FInalPlanAHATApr242017.docx 